\title{
Um Estudo sobre Turnover em Empresas de TI Embarcadas no Porto Digital de Recife
}

\author{
Cleidiane Normando Nunes ${ }^{1}$, Ivaldir de Farias Junior ${ }^{1,2}$ \\ ${ }^{1}$ Softex Recife \\ ${ }^{2}$ Universidade de Pernambuco - UPE \\ apoio@next.org.br, ivaldir.farias@upe.br
}

\begin{abstract}
Resumo. A gestão de pessoas no mundo contemporâneo vem passando por diversas mudanças, desde o avanço tecnológico que modifica a forma de trabalho de diversos cargos, bem como o acesso rápido as informações. Além disso, existe a competitividade global proporcionada pela internet em busca de novos talentos ou especialistas em determinadas áreas de conhecimento. Ou seja, globalização pode ser entendida como um processo econômico e social que estabeleceu uma integração entre os países e as pessoas do mundo. Neste sentido, o objetivo geral desta pesquisa é reunir boas práticas que auxiliem na redução do turnover dos colaboradores nas organizações. Para alcançar este objetivo, a metodologia utilizada nesta pesquisa foi uma revisão de literatura em conjunto com uma pesquisa qualitativa com 46 gestores pertencentes a 43 empresas embarcadas no Porto Digital. O instrumento de coleta utilizado foi um questionário online semiestruturado com perguntas abertas e fechadas. Com base nos resultados obtidos dos participantes, espera-se contribuir para uma melhor gestão de pessoas dentro das organizações através da explicitação das boas práticas sobre turnover recomendadas pela pesquisa em voga.
\end{abstract}

\section{Introdução}

Os avanços tecnológicos têm exigido cada vez mais profissionais bem preparados (ANG; SLAUGHTER, 2004) e como reflexo destes avanços o capital humano ganha uma maior visibilidade e se torna essencial para o crescimento e desenvolvimento das organizações (PELOSO; YONEMOTO, 2010). De acordo com Trindade (2007), com a globalização as empresas passaram a preocupar-se não apenas com os concorrentes e as exigências do mercado, mas também em dar ênfase às pessoas. Segundo o referido autor, exige-se que o modelo tradicional de gestão do capital humano seja modificado, dando lugar as práticas e políticas que facilitem a atração e retenção deste capital.

Segundo a Federação das Associações das Empresas Brasileiras de Tecnologia da Informação (ASSESPRO), o setor tecnológico é um dos mais impactados pelo turnover. "O Turnover, também conhecido como rotatividade de pessoal, é o movimento de entrada e saída de funcionários em uma empresa, demonstrado por meio de um índice de rotatividade" (FELPS, 2009). Anualmente a ASSESPRO elabora um estudo que demonstra o índice de turnover em todo o setor tecnológico do país (ASSESPRO, 2019). 
Para Gomes e Silva (2014) as empresas do segmento de Tecnologia da Informação (TI), concentram seus esforços para acompanharem as tendências do mercado e permanecerem competitivas. De acordo com Josko (2004) ter profissionais envolvidos e que detenham conhecimento diferenciado, é necessário para manter e até aumentar a competitividade de uma organização. Porém as falhas na gestão desses colaboradores e a grande demanda de mão de obra na área influenciam na migração dos profissionais de uma organização para outra (ANG; SLAUGHTER, 2004).

O turnover impacta não só no andamento dos projetos da empresa, mas retira da mesma um percentual significativo do lucro esperado (FELPS et al., 2009). Diante destas informações, o estudo é norteado pela seguinte pergunta de pesquisa: Quais são as boas práticas para auxiliar na minimização do turnover nas micro e pequenas empresas embarcadas no Porto Digital?

\section{Turnover}

De acordo com Abbasi; Hollman (2000) turnover é a movimentação de trabalhadores entre as empresas. Por outro lado, Lucena (1995) considera que o turnover é uma forma de renovar o "sangue" da empresa, especialmente em funções chaves e críticas. O autor Mobley, (1992, p. 31) defende que os profissionais que ocupam cargos na alta gestão devem ter a capacidade de fazer um diagnóstico prévio da natureza e de fatores determinantes do turnover. Para o mesmo autor é necessário avaliar as consequências negativas e positivas, criar programas, práticas e políticas para tratar o fenômeno, avaliar as mudanças e se antecipar quanto aos efeitos causados pelo turnover.

De acordo com a Assespro (2016) é de extrema importância que uma empresa tenha conhecimento sobre seus índices de turnover, pois eles podem mostrar como anda sua saúde organizacional. Para Borges; Ramos (2013, p. 5) "quando o índice de turnover de uma organização está elevado, significa que algo não vai bem e necessita de melhorias".

\subsection{Causas do turnover nas empresas}

Os profissionais têm buscado cada vez mais trabalhar em empresas que satisfaçam suas expectativas e seus desejos profissionais. Sobretudo os mais jovens, têm feito uma avaliação cautelosa nas empresas em que pretendem trabalhar, e como consequência disto os índices de turnover nas organizações tem se elevado quando esses jovens não tem a expectativa desejada satisfeita.

Chiavenato (2002) define fluxo de entrada e saída de colaboradores de uma empresa como turnover. Marras (2000) conceitua o turnover como "o número de empregados desligados da empresa em um determinado período comparativamente ao quadro médio de efetivos". Segundo Chiavenato (2002) o turnover não é apenas uma causa, mas também um efeito e consequência, de fenômenos externos e internos que influenciam nas atitudes e comportamentos dos colaboradores.

De acordo com Calisir et. al (2011) os seguintes motivos influenciam na decisão de um colaborador de deixar a empresa: falta de perspectivas e impossibilidade ou muita dificuldade de ascensão, o clima organizacional negativo, atividades não compatíveis com a remuneração, falta de políticas de reconhecimento e exigências de metas inalcançáveis. 
Já Souza (2003), aponta outros fatores como: condições físicas e ambientais de trabalho inadequadas, desvio do foco de motivação pessoal e falta de política salarial e de benefícios. Mas uma das principais causas apontadas pelo autor é a subjugação do profissional às condições de trabalho precárias e desgastantes. Outro fator que eleva a taxa de turnover é a baixa remuneração, de acordo com o Serviço Brasileiro de Apoio às Micro e Pequenas Empresas publicado no ano de 2016.

\section{Metodologia}

Nessa seção apresentamos o plano de pesquisa, definido para realização do estudo com gestores de empresas de tecnologia. Para tanto foi adotada como instrumento de coleta a aplicação de um questionário online semiestruturado com 19 questões. O estudo foi concentrado nos gestores de TI a fim de identificar as melhores práticas para a retenção de talentos nem micro, pequena e médias empresas de tecnologia embarcadas no Porto Digital. O estudo foi dividido em 1uatro fases, são elas:

Fase 1 (Estudo da base teórica): nesta fase o principal foco foi aprofundar os conhecimentos sobre turnover evidenciando o que a literatura tem de mais atual sobre o tema em voga. E por meio dela obtivemos informações para delinear e refinar a pesquisa auxiliando na elaboração do instrumento de coleta (questionário).

Fase 2 (Survey): nesta fase foi desenvolvido e aplicado o questionário a gestores de micro e pequenas empresas do Porto Digital. Este questionário visou levantar dados para avaliar e identificar boas práticas para diminuir o turnover nestas empresas.

Fase 3 (Resultados): Nesta fase foi realizada a análise sobre os dados coletados na fase 2. Para analisar os dados utilizamos o Excel para auxiliar na análise do conteúdo.

Fase 4 (Boas práticas para a redução do turnover nas micro e pequenas empresas embarcadas no Porto Digital): Nesta última fase, foi identificado um conjunto de boas práticas, que contemplam informações obtidas na análise através dos dados extraídos na pesquisa junto aos gestores.

\section{Resultados}

Participaram da pesquisa 46 gestores de diversos cargos pertencentes a 43 empresas embarcadas no Porto Digital. Vale salientar que todos os gestores participantes desta pesquisa responderam integralmente ao questionário online.

Com relação à titulação, percebe-se que o nível de gestores graduados e especializados são os maiores sendo $(43,5 \%)$ para graduação e $(34,8 \%)$ para especialização, já os gestores com titulação de mestre $(15,2 \%)$ para doutor $(4,3 \%)$, os respondentes de nível técnico formavam a apenas $(2,2 \%)$ do total.

Quando perguntados se sentiam motivados em suas funções, $(45,7 \%)$ se consideravam muito motivados, $(43,5 \%)$ se sentiam somente motivados e apenas (10,9\%) consideravam-se pouco motivados. Entre os respondentes que se consideravam pouco motivados, existiam cargos como líder técnico, supervisor, gerente e coordenador, ou seja, não são cargos operacionais.

Sobre as empresas dos quais os respondentes da pesquisa pertenciam, $(23,9 \%)$ são de microempresas, $(54,3 \%)$ são de pequeno porte, $(8,7 \%)$ são de médio porte, e por fím, 
(13\%) são de grande porte. É importante afirmar que dessas empresas, somente $(21,7 \%)$ possuem um departamento que cuida de seu capital humano.

Foi constado que apenas $(23,9 \%)$ das empresas entrevistadas adotam o plano de carreiras. Verificamos ainda que este método de gestão é utilizado por empresas de diversos portes (pequeno, médio e grande), no entanto o número mais expressivo nas respostas "SIM" é das empresas de maior porte.

Analisando os índices de turnover de funcionários das empresas entrevistadas, percebemos que $(78,3 \%)$ dos gestores consideram que suas empresas têm um nível baixo de entrada e saída de funcionários, $(17,4 \%)$ consideram mediano e apenas $(4,3 \%)$ tem uma alta turnover.

Quanto aos possíveis motivos do turnover nas organizações, foram sugeridos pelos entrevistados os seguintes motivos:

- Relacionamento com a família; Falta de política salarial; Trabalho repetitivo; Relações negativas com os demais colaboradores das empresas; Estilo gerencial inadequado; Ambiente de trabalho inadequado; Sobrecarga de trabalho; Falha na comunicação; Expectativas profissionais não alcançadas; Fatores ambientais e culturais.

Ao questionar os gestores sobre os principais fatores que levam o colaborador a trocar de empresa, obtivemos as seguintes proporções: expectativas profissionais não alcançadas com (19,9\%), estilo gerencial (14\%), ambiente de trabalho e relação com os demais colaboradores $(11,7 \%)$, falta de política organizacional para os colaboradores $(11,1 \%)$, falha na comunicação $(8,8 \%)$, sobrecarga de trabalho $(7,6 \%)$, trabalho repetitivo $(5,8 \%)$, Relacionamento com a família $(3,5 \%)$, fatores ambientais $(2,9 \%)$, propostas do mercado $(1,8 \%)$.

O resultado encontrado é convergente com pesquisa divulgada na matéria da revista Exame (2014), cuja principal razão apontada para a mudança de emprego é a falta de oportunidade de crescimento na empresa atual.

Quando perguntados sobre os tipos de benefícios oferecidos aos colaboradores, obtiveram-se as seguintes respostas: $(27,2 \%)$ plano de saúde, $(23,1 \%)$ vale alimentação, $(18,9 \%)$ auxílio creche, $(9,5 \%)$ ajuda de custo com cursos, (6,5\%) participação nos lucros, $(3 \%)$ auxílio farmácia e $(1,8 \%)$ foram mencionados também auxílios como participação nos lucros, que é plano de Stock Option.

\subsection{Boas práticas utilizadas para a retenção dos colaboradores}

Quanto às boas práticas utilizadas pelos gestores para a retenção de seus colaboradores, e consequentemente, diminuir o turnover em suas organizações, é possível perceber a preocupação com o ambiente de trabalho que nesta pesquisa foi considerada a prática mais utilizada pelos entrevistados. Essa teve um índice de $(22,4 \%)$. Ainda segundo os entrevistados, uma forma utilizada para manter os colaboradores por mais tempo nas organizações é manter a sinceridade e a transparência organizacional $(17,9 \%)$. A pesquisa também revela que a remuneração também é utilizada pelo gestor para influenciar os colaboradores a permanecerem na organização $(16,4 \%)$. Vale salientar que foram citados ainda categorias como oferecer bons benefícios (3\%), oferecer crescimento profissional (3\%), proporcionar desafios as 
aos colaboradores $(4,5 \%)$, reconhecimento e capacitação, ambas sendo citadas por $(6 \%)$ dos gestores, estilo gerencial e autonomia também estão no mesmo patamar segundo os respondentes com $(9 \%)$.

Uma categoria nos chamou a atenção que foi o alinhamento do perfil profissional as atividades executadas, a mesma foi citada por (3\%) dos respondentes e segundo um deles adequar o perfil do colaborador a realidade da empresa auxiliou na redução do turnover em sua organização. Abaixo trecho da fala do respondente B:

"Diminuímos consideravelmente nossa turnover selecionando as pessoas com perfil compativel com nossa realidade”.

Foram apontados os principais desafios enfrentados pelos gestores para reter seus talentos. O maior desafio enfrentado pelos gestores de micro e pequenas empresa do segmento tecnológico de Pernambuco é manter seus colaboradores motivados (33,9\%). Mayo (2003) afirma que o fator motivação é um grande influenciador na retenção de talentos, pois através da motivação consegue-se descobrir e desenvolver o potencial de uma pessoa.

Os mesmos autores dizem ainda que: "Motivar quer dizer mover para a ação, mobilizar energias e esforços na busca da realização de determinada direção".

Diante deste contexto, e como principal resultado da pesquisa, foram reunidas boas práticas para auxiliar na diminuição do turnover em micro e pequenas empresas embarcadas no Porto Digital, com base em pesquisas bibliográficas e estudos de campo/empírico com gestores de empresas de tecnologia. As boas práticas (Tabela 1) têm como objetivo, contribuir e incentivar os gestores a buscarem novos métodos para reter seus talentos nas organizações.

Tabela 1 - Legenda de identificação das Práticas

\begin{tabular}{c|c|c|c}
\hline Legenda & Descrição das práticas & Revisão literária & $\begin{array}{c}\text { Respondente } \\
\text { (Survey) }\end{array}$ \\
\hline P1 -Prática 1 & Criar um estilo de gestão transparente. & $\begin{array}{c}\text { (HERMAN, 1993; } \\
\text { FELPS, 2009) }\end{array}$ & $\begin{array}{c}\text { Resp. 1, 2, 3, 5, 6, 7 } \\
\text { e 8. }\end{array}$ \\
\hline P2- Prática 2 & $\begin{array}{c}\text { Promover um ambiente de trabalho, estimulante } \\
\text { e confortável aos colaboradores. }\end{array}$ & $\begin{array}{c}\text { (CARLOS, 2010), } \\
\text { ARAÚJO (2009) }\end{array}$ & $\begin{array}{c}\text { Resp. 2, 7, 10, 11, } \\
14,15 \text { e 16 }\end{array}$ \\
\hline P3 - Prática 3 & Oferecer salários atrativos. & $\begin{array}{c}\text { (SEBRAE, 2016), } \\
\text { MONTEIRO (2012) }\end{array}$ & $\begin{array}{c}\text { Resp. 2, 17, 18, 19 e } \\
20 .\end{array}$ \\
\hline P4 - Prática 4 & Aplicar o capital humano de forma adequada. & $\begin{array}{c}\text { (CHIAVENATO, } \\
\text { 2002) }\end{array}$ & Resp. 21 e 27 \\
\hline P5 - Prática 5 & Desenvolver um departamento de RH & $\begin{array}{c}\text { (SILVA, 2006; } \\
\text { FELPS, 2009) }\end{array}$ & Resp. 22 e 23 \\
\hline P6 - Prática 6 & Criar planos de desenvolvimento de carreiras. & $\begin{array}{c}\text { BOHLANDER et al. } \\
\text { (2003) ARAÚJO } \\
\text { (2009) }\end{array}$ & $\begin{array}{r}\text { Resp. 17 } \\
\text { MONTEIRO (2012) }\end{array}$ \\
\hline P7 - Prática 7 & Elaborar práticas de valorização e \\
reconhecimento. & $\begin{array}{c}\text { DIAS, (2017); } \\
\text { (SEBRAE, 2016) }\end{array}$ & $\begin{array}{c}\text { Resp. 6, 24, 25 e 26 } \\
\text { e 29 }\end{array}$
\end{tabular}




\begin{tabular}{c|c|c|c} 
P8 - Prática 8 & Incentivar e ofertar capacitação e treinamento. & $\begin{array}{c}\text { CHIAVENATO } \\
(2002)\end{array}$ & $\begin{array}{c}\text { Resp. 18, 24, 27, 28 } \\
\text { e 29 }\end{array}$ \\
\hline P9 - Prática 9 & Proporcionar benefícios diversificados. & DIAS, (2017) & Resp. 25
\end{tabular}

\section{Considerações Finais}

Os índices de turnover nas organizações estão diretamente relacionados à evolução do perfil profissional e do conceito de valorização do capital humano. Para controlar este fenômeno, é necessário que se adote boas práticas. Considerando este aspecto, a pesquisa evidencia que muitas empresas (78\%) não dispõem departamento de $\mathrm{RH}$, mas que a grande maioria executa ações para manter seus talentos. Somente $23,9 \%$ das empresas respondentes tem formalmente um plano de carreira. Isso ocasiona aos empregados uma baixa expectativa de crescimento profissional. Essa baixa expectativa, segundo a pesquisa representa é um dos principais elementos causadores de rotatividade nas empresas de TI embarcada no Porto Digital. Obviamente que existem outros vários motivos que são determinantes para o turnover. Segundo a pesquisa podemos citar: Relacionamento com a família; Falta de política salarial; Trabalho repetitivo; Relações negativas com os demais colaboradores das empresas; Estilo gerencial inadequado; Ambiente de trabalho inadequado; Sobrecarga de trabalho; Falha na comunicação e Fatores ambientais e culturais.

Neste sentido, foi de suma importância a identificação dos fatores determinantes que levam os colaboradores a trocarem de empresa que consequentemente deixam uma lacuna de capital intelectual dentro das empresas. Por isso essa pesquisa é relevante, e tem como principal contribuição a reunião de um conjunto de boas práticas que objetivam auxiliar as empresas a diminuírem esse turnover.

Como trabalhos futuros espera-se que as práticas propostas nesta pesquisa sejam implementadas em um cenário real para fazer a homologação de sua eficiência e eficácia.

\section{REFERÊNCIAS}

ARAUJO, Luis César G de. Gestão de Pessoas - Estratégias e Integração Organizacional. 2. ed. São Paulo: Atlas, 2009.

ABBASI, S.M.; HOLLMAN, K. W. Turnover: The real bottom line. Public Personnel Management, v.29, n.3, 2000.

ANG, S. and Slaughter, S. (2004) Turnover of information technology professionals: the effects of internal labor market strategies. ACM SIGMIS Database, v. 35, n. 3, p. 1127.

ASSESPRO. RETENÇÃO DE TALENTOS É UM DOS MAIORES DESAFIOS DA TI EM 2019. Disponível em: http://www.assespro-rs.org.br/retencao-de-talentos-e-umdos-maiores-desafios-da-ti-em-2019/ Acessado em 23 de janeiro de 2019.

BORGES, Mara Santos; RAMOS, Noézia Maria. Turnover: uma consequência de estratégias ineficientes de gestão empresarial. VIII Convibra, 2011. 
BOHLANDER, George W. et al. Administração de Recursos Humanos. São Paulo: Pioneira Thomson Learning, 2003.

CALISIR, F.; GUMUSSOY, C.A.; ISKIN, I. Factors affecting intention to quit among IT professionals in Turkey. Personal Review, v.40, n. p. 514-533, 2011.

CARLOS, Rafael Luiz Alfaia, 2010 Disponivel em: http://www.administradores.com.br/artigos/carreira/o-ambiente-de-trabalhocomomotivacao-e-diferencial-entre-empresas/50309/ acesso em 26/06/2018.

CHIAVENATO, I. Recursos humanos: ed. Compacta, 7 ed. São Paulo: Atlas, 2002.

DIAS, E. https://www.dicionariofinanceiro.com/turnover/ acesso em 06/05/2019

FELPS, Will et al. Turnover contagion: How coworkers' job embeddedness and job search behaviors influence quitting. Academy of Management Journal, v. 52, n. 3, p. 545-561, 2009.

GOMES, C.F. S; SILVA, R.A. Avaliações das práticas de gestão de pessoas no segmento de tecnologia da informação (TI). 2014.

HERMAN, R. Como manter os bons funcionários: estratégias para solucionar o grande problema de recursos humanos da década. São Paulo: Marron Books, 1993.

JOSKO, João Marcelo Borovina et al. Gestão de pessoas em tecnologia da informação: uma visão perspectiva das abordagens. 2004.

LUCENA, Maria Diva da salete. Planejamento de recursos humanos. São Paulo: Atlas, 1995.

MAYO, Andrew. O valor humano da empresa: valorização das pessoas como ativos. São Paulo: Pearson Prentice Hall, 2003.

MARRAS, J.P. Administração de recursos humanos: do operacional ao estratégico. São Paulo: Futura, 2000.

MOBLEY, William H. Turnover: Causas, consequências e controle; tradução de Vânia Conde, Porto Alegre: Ortiz, 1992. Disponível em: http://www.convibra.com.br/upload/paper/adm/adm_2784.pdf acesso em $12 / 02 / 2018$.

MONTEIRO, Luiz Eduardo dos Santos. Importância do plano de carreira e remuneração para a redução do turnover dentro das empresas: caso ELETROBRAS. 2012. Tese de Doutorado.

PELOSO, Ayslan Cavalcante; YONEMOTO, Hiroshi Wilson. Atração, desenvolvimento e retenção de talentos. ETIC-ENCONTRO DE INICIAÇÃO CIENTÍFICA-ISSN 21-76-8498, v. 6, n. 6, 2010.

SEBRAE. Rotatividade de colaboradores. Disponível em: http://www.sebrae.com.br/sites/PortalSebrae/ acessado em 26 de março de 2019.

SILVA, Elson Magno da. Os efeitos da liderança na retenção de talentos um estudo sobre comprometimento e turnover numa indústria petroquímica. 2006. Tese de Doutorado. Dissertação (Mestrado em Administração)-IBMEC. Rio de Janeiro. 
SOUZA, R. M. Retenção de Talentos e sua Importância na Gestão de Recursos Humanos. 2011. 29 f. Monografia (Especialização em Recursos Humanos - Gestão de Pessoas e Competências). Centro Universitário Filadélfia, Unifil. Londrina, Pr., 2011.

TRINDADE, P. S. S. Retenção de Talentos. Porto Alegre, 2007. 